\title{
CRITERIA OF DYNAMICAL ISOLATION OF BINARIES AND
}

\section{MULTIPLES}

\author{
LUDMILA KISELEVA \\ Institute of Astronomy, Madingley Rd., Cambridge CB3 OHA, \\ $U K$
}

AND

JOANNA ANOSOVA

National Astronomical Observatory, 2-21-1 Osawa, Mitaka, Tokyo 181, Japan

In order to obtain an objective criterion for dynamical isolation of binaries within systems of large multiplicity we study numerically the dynamical behavior and average parameters of stable hierarchical triple systems containing a close binary. Using the new criterion for hierarchical stability of triple systems with different mass ratios of components (Kiseleva, Eggleton, Anosova 1994; Kiseleva, Eggleton, Orlov 1994) the perturbing force from the outer body on the close inner binary is estimated. On this basis, the critical separations are obtained when both inner and outer orbits are practically not perturbed. Because the dispersion of masses has a very strong influence on dynamical processes in $\mathrm{N}$-body systems, mass ratios of subsystems, and sometimes within subsystems, should always be taken into account. Anosova and Orlov (1989) and Anosova (1994) proposed a parameter of isolation of close pair $i, j$ from its nearest neighbor

$$
q_{b}=\left(\frac{m_{i}+m_{j}}{2 m_{l}}\right)^{1 / 3} \frac{r_{l}}{r_{i j}}>q_{b}^{*}
$$

where $l$ denotes the closest body besides $\mathrm{i}, \mathrm{j}(l=1,2 \ldots N ; l \neq i, l \neq j), r_{i} j$ is the separation between the components of the pair, $r_{l}$ is the distance of the body $l$ from the center mass of the pair i,j and $q_{b}^{*}$ is an adopted critical value of $q_{b}$. This parameter $q_{b}$ is the root cube of the ratio of of perturbing accelerations of one of the binary's components $i$ or $j$ from the other component and from the perturber $l . q_{b}$ is a convenient parameter to use because it includes the mass ratio of tested subsystems. For considered 
mass rations of close and wide binaries the value of $q_{b}$ is between 2.7 and 8.4 .

The averages in time of values of orbital parameters for close and wide binaries for stable nearly unperturbed cases are calculated and the corresponding approximations are then made. The obtained criterion of isolation can be generalized for subsystems with larger multiplicities and also applied to multiples within clusters.

\section{References}

Anosova, J.P., Orlov, V.V. Astrophys. Space Sci.,1989, 161,209

Anosova, J.P. Astrophys. Space SCi., 1994, submitted.

Kiseleva, L.G., Eggleton, P.P., Anosova, J.P. (1994) Mon. Not. R. Astr. Soc.,1994, 267, 161.

Kiseleva, L.G., Eggleton, P.P., Orlov, V.V.(1994) Mon. Not. R. Astr. Soc., 1994, in press 